\title{
Colorectal Cancer Surgery During the COVID-19 Pandemic: A Single Center Experience
}

\author{
DAVIDE PERTILE ${ }^{1}$, MARCO GIPPONI $^{2}$, ALESSANDRA APRILE ${ }^{1}$, \\ PAOLA BATISTOTTI ${ }^{1}$, CAROL MARZIA FERRARI $^{1}$, ANDREA MASSOBRIO ${ }^{1}$, \\ DOMENICO SORIERO ${ }^{1}$, LORENZO EPIS ${ }^{1}$ and STEFANO SCABINI ${ }^{1}$ \\ ${ }^{1}$ General and Oncologic Surgery, IRCCS Ospedale Policlinico San Martino, Genoa, Italy; \\ ${ }^{2}$ Breast Surgery Clinic, IRCCS Ospedale Policlinico San Martino, Genoa, Italy
}

\begin{abstract}
Background/Aim: A notable re-allocation of healthcare resources and specific clinical and organizational measures have been required to prevent COVID-19 infection among hospitalized patients and healthcare workers. Patients and Methods: From March 9th to May 9th 2020 we performed colorectal cancer elective surgery on 25 patients: a pre-hospital screening was carried out in order to avoid hospitalization of patients suspected of COVID-19 infection. Results: All patients (median age $=76$ years; range $=37-88$ years) were considered suitable for admission after telephone triage; the median interval between primary diagnosis and hospital admission was 23.1 days (range $=1$ 55 days). The median hospitalization was 7.8 days (range $=4-18$ days). One COVID-19-associated death was reported. Conclusion: Our experience demonstrates that safe colorectal cancer elective surgery can be performed during the pandemic COVID-19. Further consensus and guidelines to prevent diffusion of pandemic diseases among hospitalized patients and healthcare workers still need to be implemented.
\end{abstract}

By the end of February 2020, the first patient with severe acute respiratory syndrome coronavirus 2 (SARS-CoV-2) was diagnosed and, thereafter, almost 225,000 new infections were detected in Italy, with approximately 32,000 deaths. In our region, Liguria, 9,159 COVID-19 patients were identified, and 1,355 died by May 17th $2020(1,2)$. This COVID-19 pandemic has required a notable re-allocation of

This article is freely accessible online.

Correspondence to: Davide Pertile, General and Oncologic Surgery, IRCCS Ospedale Policlinico San Martino, L.go R. Benzi 10, 16132 Genoa, Italy. Tel: +39 0105553216, Fax: +39 0105556727, e-mail: davide_pertile@libero.it

Key Words: Colorectal cancer, COVID 19, healthcare, infections, prevention, colorectal surgery. healthcare resources, with an immediate re-organization of all clinical activities, including Colorectal Cancer Units. Elective Surgical departments have been forced to change their usual clinical practices to contribute to the growing need for healthcare resources during this state of emergency (3).

Here we report a retrospective clinical study performed at the Surgical Oncological Unit of our Policlinic Hospital, which assessed a patient-tailored program with two objectives: i) to avoid the hospitalization of COVID-19positive patients by means of pre- and in-hospital screening procedures in order to protect patients as well as Healthcare Workers (HCWs) from any undue risk of exposure to SARS$\mathrm{CoV}-2$, and ii) to define the clinical pictures which need a priority surgical treatment.

\section{Patients and Methods}

Specific consent about privacy and data protection was required.

Patient screening flowchart. A specific flowchart was developed in order to screen patients for suspected COVID-19 symptoms (fever, cough, sore throat, shortness of breath and other respiratory symptoms). This flowchart was adopted in three different settings: i) pre-hospital, ii) in-hospital and iii) post-discharge (Figures 1) (47), according to national legislation ISS COVID-19 n. 1/2020 and the recommendations provided by the WorId Health Organization on February 27th 2020 , and suitably modified to meet our local requirements $(8-11)$.

Telephone triage. A dedicated nurse, properly trained by the Hospital Infection Control Team, called the patient 7 days before hospitalization, filling out a specific anamnestic questionnaire. Moreover, patients were kindly asked to contact the Colorectal Department if they developed new symptoms during the interval period.

The questions used are shown in Table I, and COVID-19 symptoms were classified according to major and minor criteria shown in Table II. Patients found positive at one major or two or more minor criteria were reported to the Public Health Office, followed by activation of home monitoring and temporary suspension of surgery. A new phone call was performed 2 weeks later and if the patient was perceived as negative after answering 


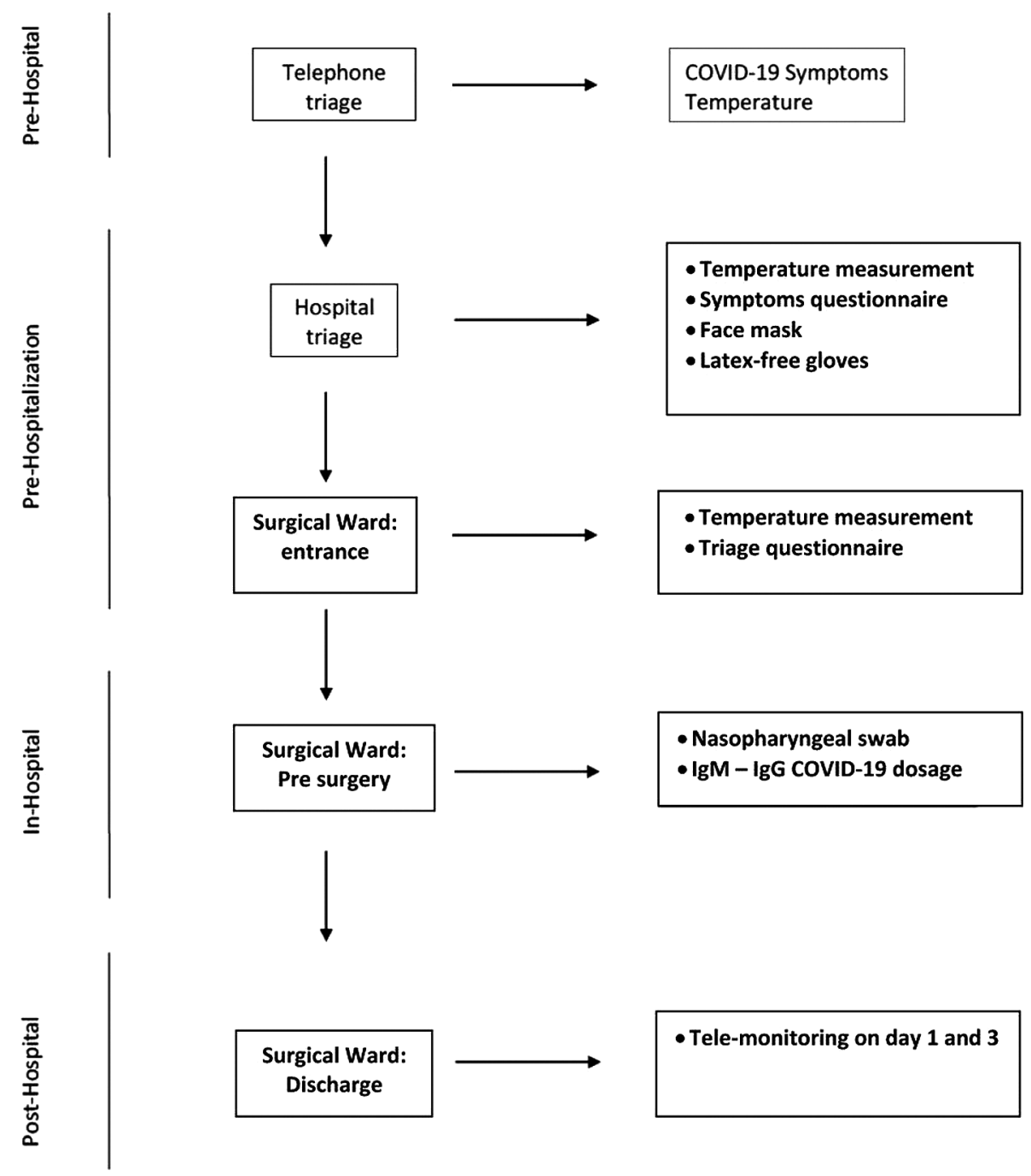

Figure 1. Outline of pre-, in-, and post-hospital selection procedures. (Adapted from 25).

the questionnaire, a telephone triage was repeated one day before hospitalization.

In-hospital triage. On arrival at the surgical ward, patients followed a standardized procedure before any interaction with $\mathrm{HCWs}$, which involved: i) use of an automatic hand sanitizer dispenser; ii) use of a disposable face mask from an automatic dispenser, and iii) use of disposable latex-free gloves (12-15). The nurse allocated to the inhospital triage was equipped with Personal Protective Equipment (PPE), i.e.: gloves, face mask, gown and goggles. Patients were given a COVID-19 questionnaire prepared by the Hospital's Department of Public Health Epidemiology, which included clinical health data, such as body temperature and any COVID-19 symptoms present during the previous two weeks. An infrared noncontact thermometer was used to determine body temperature: only patients with temperature lower than $37.5^{\circ} \mathrm{C}$ were admitted to the Surgical ward. Visits from relatives during hospitalization were not allowed and patients with cognitive and motor disability were assisted by healthcare workers. The Surgical Unit Chief personally called every day one relative of each patient providing information about their clinical course. Following admission, patients were immediately subjected to nasopharyngeal (NP) swab, followed by molecular testing using real-time reverse transcriptase PCR (RTPCR) for the detection of COVID-specific genes: i) RdRP, ii) E, iii) $\mathrm{N}$ and iv) $\mathrm{S}$, as well as humoral tests for IgM and IgG COVID-19 antibodies. While waiting for the final results, they were kept in isolation in a single room. Surgery was scheduled when the NP swab molecular test came negative, while the COVID-19 serology test was carried out only for investigational purposes without affecting the scheduled operation. Upon completion of the surgical procedure, the surgeon informed the patient's family members of the immediate surgical outcome because visitors weren't permitted to access to the ward. Patients were discharged once they were clinically stable to minimize the risk of re-admission. Periodic information over the phone was ensured with the referring person of each patient. 
Table I. Clinical assessment check-list [adapted from (25)].

\begin{tabular}{ll}
\hline Symptoms & Medical history \\
\hline Fever & Do you live with a patient confirmed as having COVID-19? \\
Cough & Did you have physical contact with a patient confirmed as having COVID-19? \\
Dyspnea & Did you have physical non-protected contact with secretion from a person with COVID-19? \\
& Did you have prolonged contact (face to face, more than 15 min and less than 2 m distance) with a confirmed patient? \\
& Did you have prolonged contact in closed spaces (elevator, meeting room, more than 15 min and less than 2 m distance) \\
& with a patient with COVID-19? \\
& If you are a Healthcare Worker: Did you have any contact without proper PPE with a confirmed patient or with infected biological fluid? \\
& Have you traveled in the past 2 weeks with a confirmed COVID-19-positive patient?
\end{tabular}

Post-discharge triage. A specifically allocated, well-trained nurse called each patient at home on the first and third days following discharge to check on their clinical status (body temperature, pain level utilizing a Visual Analogue Scale, physiologic functions, etc.). On the seventh post-operative day patients could visit the Outpatient Clinic for the first time. In general, post-discharge visits were reduced to the minimum required (15). In case a patient developed COVID-19-related symptoms, Public Health Officers and Family Doctors were informed to check the clinical course of this patient.

Patient selection. According to the COVID-19 Guidelines for Triage of Colorectal Cancer Patients in the Semi-Urgent Setting, patients with the following disease presentation were selected for surgery (16): i) nearly obstructing colon cancer, ii) nearly obstructing rectal cancer, iii) cancer requiring frequent transfusions, iv) asymptomatic colon cancer, v) rectal cancer after neoadjuvant chemo radiation with no response to therapy, vi) cancers with concern about local perforation and sepsis, vii) earlystage rectal cancer where adjuvant therapy would not be appropriate.

Diagnoses that could be deferred to 3 months included: i) malignant polyps, either with or without prior endoscopic resection, ii) prophylactic indications for hereditary conditions, iii) large, benign appearing asymptomatic polyps, iv) small, asymptomatic colon carcinoids, and v) small, asymptomatic rectal carcinoids.

Alternative treatment approaches were considered to delay surgery. For locally advanced resectable colon cancer neoadjuvant chemotherapy was considered for 2-3 months followed by surgery. For rectal cancer cases with clear and early evidence of downstaging from neoadjuvant chemo radiation, surgery was delayed if: i) additional waiting time was safe, and ii) additional chemotherapy could be administered. Delays in surgery for locally advanced rectal cancer or recurrent rectal cancer requiring exenterative surgery were agreed if additional chemotherapy could be administered, while delaying surgery in oligometastatic disease was agreed if effective systemic therapy was available.

All surgery-eligible patients were assessed weekly by a multidisciplinary team using a clinical report, including information about i) diagnosis, staging, and priority, such as the curative intent (surgery with palliative intent was not allowed), ii) recent staging, iii) symptomatic tumor, high malignancy neoplasm or iv) cases of advanced stage disease, where neoadjuvant therapy could not be proposed or had been already performed; ii) co-morbidity factors and American Society of Anesthesiologists (ASA) score; iii) anamnestic questionnaire on self-isolation at home, exposure of close family members, presence of symptoms, etc.
Table II. Major and minor criteria for the assessment of COVID-19related symptoms.

\begin{tabular}{lc}
\hline Major criteria & Minor criteria \\
\hline Fever & Headache or dizziness \\
Cough & Diarrhea \\
Dyspnea & Nausea and vomiting \\
Myalgia or fatigue & \\
Expectoration & \\
\hline
\end{tabular}

Table III. Patient selection based on preoperative diagnosis.

\begin{tabular}{ll}
\hline Criteria & Frequency, n (\%) \\
\hline $\begin{array}{l}\text { Malignant polyp, non-amenable } \\
\text { to endoscopic resection }\end{array}$ & $3(12.0 \%)$ \\
Neoadjuvant treatment & $4(16.0 \%)$ \\
Nearly obstructing colon cancer & $6(24.0 \%)$ \\
Rectal cancer (stage I) & $1(4.0 \%)$ \\
Nearly obstructing rectal cancer & $9(36.0 \%)$ \\
Neuroendocrine rectal tumor & $1(4.0 \%)$ \\
Locoregional rectal recurrence & $1(4.0 \%)$ \\
\hline Total & $25(100.0 \%)$ \\
\hline
\end{tabular}

\section{Results}

Overall, from March 9th to May $9^{\text {th }} 2020,25$ patients (median age $=76$ years; range $=37-88$ years) underwent telephone triage by a specifically allocated Nurse of the General and Oncologic Surgery at Ospedale Policlinico San Martino in Genoa, all of whom were considered suitable for admission. The preoperative diagnosis and the type of surgical procedure are reported in Tables III and IV. The median interval between primary diagnosis and hospital admission was 23.1 days (range $=1-55$ days). With regard to 
Table IV. Type of surgical procedure stratified by preoperative histology.

Procedure

Video-laparoscopic right hemicolectomy

Open right hemicolectomy

Right hemicolectomy with right posterior and atypical right posterior liver resection

Right hemicolectomy with atypical liver resection (segment 8v-4a)

Right hemicolectomy with right nephrectomy

Video-laparoscopic left hemicolectomy with atypical liver resection (segment 3)

Subtotal colectomy

Robotic-assisted anterior rectal resection

Video-laparoscopic anterior rectal resection

Hartmann procedure with atypical liver resection (segment 8)

Abdomino-perineal rectal resection

Resection of rectal stump with cystectomy, small bowel resection, and lumbar-aortic lymphadenectomy
Frequency, n (\%)

$3(12.0 \%)$

$2(8.0 \%)$

$1(4.0 \%)$

$1(4.0 \%)$

$1(4.0 \%)$

$1(4.0 \%)$

$1(4.0 \%)$

$1(4.0 \%)$

$7(28.0 \%)$

$1(4.0 \%)$

$5(16.0 \%)$

$1(4.0 \%)$

Total

$25(100.0 \%)$

the postoperative course, three patients $(12 \%)$ had perineal wound dehiscence after abdomino-perineal rectal resection; one patient (4\%) had a superficial abdominal wound infection, and two others $(8 \%)$ had an intra-abdominal infection; moreover, two patients had urinary retention $(8 \%)$ (Table V). The median time of hospitalization was 7.8 days (range $=4-18$ days). At postoperative histological examination [UICC Staging System 2018 (17)], two out of 25 patients (8\%) had pTis carcinoma, five $(20 \%)$ had stage I, 8 (32\%) had stage II, $4(16 \%)$ had stage III, and $5(20 \%)$ had stage IV disease; finally, one patient had a neuroendocrine tumor.

Clinical case. A 76-year-old man with an extensive pelvic recurrence that occurred 3 years after neoadjuvant chemoradiotherapy and a Hartmann procedure for primary rectal cancer, with several comorbidity factors (chronic renal failure, bilateral ureteral stenting, hypertension, and paroxysmal atrial fibrillation) was admitted to the Emergency Department due to rectal stump bleeding with severe anemia $(\mathrm{Hb}=4.0 \mathrm{~g} / \mathrm{dl})$ and paroxysmal atrial fibrillation. At admission, molecular testing from NP swab and IgM and IgG COVID-19 antibody tests took place, all of which were negative. After preoperative stabilization of hemodynamic parameters and recovery from anemia, by means of red blood cell transfusion, the patient underwent laparotomy one week later with rectal stump resection, radical cystectomy, right ureterostomy, segmental small bowel resection, and lumbar-aortic lymphadenectomy. At histology, neoplastic recurrence of the rectal stump with bladder and small bowel involvement was detected (pT4 stage, according to the Union for International Cancer Control, with an $\mathrm{R} 0$ resection). Thereafter, he was moved to the Intensive Care Unit due to recurrent paroxysmal atrial fibrillation and, 3 days later, he returned to the surgical ward. On the sixth postoperative day, he underwent re-laparotomy due to a small bowel anastomotic leakage, and after 2 days the patient developed fever $\left(37.5^{\circ} \mathrm{C}\right)$ with dyspnea and impairment of blood saturation parameters $(\mathrm{SpO} 2=78 \%)$. On the ninth postoperative day, molecular testing proved positive although $\operatorname{IgM}$ and $\mathrm{IgG}$ COVID-19 antibody tests were still negative. Chest radiography detected diffuse interstitial thickening with bilateral focal opacifications with inflammatory features and bilateral pleural effusion. Respiratory function improved by means of oxygen mask achieving an $\mathrm{SpO} 2$ of 92\%; however, he had persistent atrial fibrillation (110 heartbeats/min) with very high pro-brain natriuretic peptide value $(24,803 \mathrm{pg} / \mathrm{ml}$; normal value $<450.00$ $\mathrm{pg} / \mathrm{ml})$. From the cardiological standpoint, the patient received intravenous diuretic (250 mg furosemide) and anti-arhythmic drugs (1.25 mg bisoprolol). Moreover, consultation from the Infectious Diseases unit suggested starting hydroxycloroquine at $400 \mathrm{mg}$ twice daily, and $80 \mathrm{mg}$ methylprednisolone plus antibiotic therapy (piperacillin/tazobactam, tigecycline, and ceftolozane/tazobactam) due to the underlying abdominal infectious complications. His clinical condition gradually improved with no sign of respiratory distress $(\mathrm{SpO} 2=98 \%)$, normal bowel and renal functions, light oral feeding, and no sign of surgical site infection, with a progressive normalization of inflammatory parameters (C-reactive protein=159 $\mathrm{mg} / \mathrm{l}$; normal value $5-10 \mathrm{mg} / \mathrm{l}$; and procalcitonin $5.59 \mathrm{ng} / \mathrm{ml}$ ) but 2 days later, he died suddenly due to cardiovascular failure.

\section{Discussion}

The COVID-19 pandemic has greatly increased and modified healthcare demands with re-allocation of healthcare resources as well as re-organization of clinical activities, including Colorectal Units. Colorectal cancer has specific disease-related features compared to other neoplastic subsets due to complications related to local disease progression, such as 
bleeding, obstruction, or perforation. Moreover, other specific therapeutic aspects are related to the possibility of a multidisciplinary approach to locally advanced stages, especially in the rectum, which may be adopted in order to avoid any delays in treatment $(18,19)$. Hence at our Surgical Oncology Clinic, a detailed algorithm was developed in line with national and international recommendations with the aim of accomplishing strict COVID-19 screening before and at the hospitalization of patients undergoing surgery for colorectal cancer. The main objective was to prevent any risks in patient survival as a result of a delay in treatment and, equally importantly, to ensure the safety of our work team.

In reality, due to the patient screening flowchart, no patient was temporarily suspended from the scheduled operation because of fever or previous hospitalization, due to COVID-19 infection. Upon pre-admission to the surgical ward, minimal patient-to-HCW interaction was enforced until a negative NP swab was obtained, which considerably reduced the risk of COVID-19 infection transmission. A possible bias of this flowchart was that some threatened by cancer diagnosis patient could minimize the presence of symptoms to expedite their operation. Nonetheless all the other preventive measures (respect of social distancing, body temperature measurement, use of PPE, isolation of patients waiting for NP results) became fundamental in preventing COVID-19 spread. In fact, no COVID-19 infection was detected among HCWs on our surgical ward during this period.

In spite of the reduced availability in operating theatres and anesthetists due to their assignment to Intensive Care Units, a more-than-relevant number of patients safely underwent colorectal surgical procedures. In one patient, however, NP swab positivity with symptoms of COVID-19 infection were detected more than 2 weeks after hospital admission. The patient was suffering from an advanced locoregional recurrence of rectal cancer that had been treated 3 years earlier primarily using neoadjuvant therapy. Moreover, he was suffering from comorbidity factors, such as severe anemia due to bleeding at the recurrence site, chronic renal failure, hypertension and paroxysmal atrial fibrillation. He underwent re-operation on the sixth postoperative day due to anastomotic leakage but, while experiencing a more than successful therapeutic response to infective complications. Unfortunately, he had a sudden cardiac failure, which justified a diagnosis of 'COVIDassociated' rather than 'COVID-related' postoperative death.

Regarding the other patients, in-hospital stay was restricted to the minimum to prevent any excessive overload of the healthcare system. During the postoperative course, patients were regularly discharged once their clinical condition was more than acceptable, and a well-designed post-discharge tele-monitoring was organized and supervised by specifically allocated nurses.
Table V. Postoperative morbidity and mortality.

\begin{tabular}{ll}
\hline & Frequency, n (\%) \\
\hline Postoperative death (COVID-associated) & $1(4.0 \%)$ \\
& \\
Postoperative complications & $3(12.0 \%)$ \\
Perineal wound dehiscence & $1(4.0 \%)$ \\
Superficial abdominal wound infection & $2(8 \%)$ \\
Deep (intra-abdominal) infection & $2(8.0 \%)$ \\
Urinary retention & $1(4.0 \%)$ \\
Hospital re-admission & \\
\hline
\end{tabular}

From a technological standpoint, as SARS-Cov-2 can potentially be spread as aerosol with $\mathrm{CO} 2$ circulation in the abdominal cavity, the use of laparoscopy in COVID-19positive patients undergoing colorectal surgery should be properly weighed. On one hand, laparoscopy might facilitate the spread of viral particles due to the positive intraabdominal pressure and the longer operative time with prolonged anesthesia; on the other, it may reduce the dissemination of aerosol compared to open surgery (20).

With regards to the therapeutic strategy during the COVID-19 pandemic in the case of locally advanced rectal cancer, short- course radiotherapy $(5 \times 5 \mathrm{~Gy})$ followed by delayed surgery (5- to 13-week interval) has, so far, led to a higher rate of severe acute post-radiation toxicity $(4.2 \%$ absolute difference) but fewer postoperative complications (13\% absolute difference), and an approximately $10 \%$ higher rate of pathological complete response $(21,22)$. Hence, a short-course regimen of preoperative radiotherapy coupled with delayed surgery might have the advantage of reducing the in-hospital length of patients' exposure to COVID-19 infection during radiotherapy with the added value of postponing surgery to a time of a more satisfactory epidemic control. Conversely, patients with clinical T4 disease might preferably have a long-course treatment, as reported in the GRECCAR-6 phase III trial, including patients with cT3/T4 or $\mathrm{TxN}+$ tumors of the mid or lower rectum who received chemoradiotherapy (45-50 Gy with 5-fluorouracil or capecitabine) and were randomly placed into groups of a 7or 11-week waiting period. This extension of four weeks after the completion of the neoadjuvant protocol had no influence on the 3-year disease-free and overall survival of those with T3/T4 rectal cancer (23). However, morbidity was significantly increased in the 11-week group compared to the 7-week group (44.5\% vs. 32\%; $p=0.0404)$ as a result of increased medical complications $(32.8 \%$ vs. $19.2 \%$; $p=0.0137$ ). In addition, these group of patients presented the worse quality of mesorectal resection (complete mesorectum $78.7 \%$ vs. $90 \% ; p=0.0156$ ) (24). 
In conclusion COVID-19 Pandemic has severely affected hospital organization and the treatment of severe diseases, such as colorectal cancer, has become far more challenging. New strategies to avoid in-hospital diffusion of COVID-19 infection have been developed in order to protect both patients and Healthcare workers. Prioritization of specific clinical features has been made necessary, due to the lack of operating rooms as well as personnel, such as anesthesiologists and nurses, who were frequently displaced to assist COVID-19 admitted patients.

Despite the difficulties during this pandemic, in our experience, colorectal cancer surgery could be carried out safely. We believe that our approach can be transferred to other clinical surgical settings, avoiding unnecessary delays of treatment. Consensus and guidelines should be provided to healthcare workers in order to support the clinical decision-making process and a proper re-allocation of healthcare resources.

\section{Conflicts of Interest}

None.

\section{Authors' Contributions}

DP: Study planning and surgical treatment; MG: Study planning and article editing; AA, PB, CMF, AM, LE and DS: surgical management and follow-up data; SS: Study Planning, surgical treatment, and article editing.

\section{References}

1 Dipartimento della Protezione civile: COVID-19 Monitoraggio della situazione. Available at: http://opendatadpc.maps. arcgis.com/apps/opsdashboard/index.html\#/b0c68bce2cce478eaa c82fe3 8d4138b1 [Last accessed on December 10 2020]

2 Coronavirus: 1'aggiornamento sulla situazione in Liguria - Alisa. Available at: https://www.alisa.liguria.it [Last accessed on April 30 2020]

3 Hübner M, Zingg T, Martin D, Eckert P and Demartines N: Surgery for non-Covid-19 patients during the pandemic. PLoS One 15(10): e0241331, 2020. PMID: 33095834. DOI: 10.1371/ journal.pone. 0241331

4 European Centre for Disease Prevention and Control; Cluster of pneumonia cases caused by a novel coronavirus, Wuhan, China 2020. Available at: https://www.ecdc.europa.eu/sites/default/ files/documents/Risk-assessment-pneumonia-Wuhan-China-22Jan-2020.pdf [Last accessed on April 30 2020]

5 Zhou F, Yu T, Du R, Fan G, Liu Y, Liu Z, Xiang J, Wang Y, Song B, Gu X, Guan L, Wei Y, Li H, Wu X, Xu J, Tu S, Zhang $\mathrm{Y}$, Chen $\mathrm{H}$ and Cao B: Clinical course and risk factors for mortality of adult inpatients with COVID-19 in Wuhan, China: A retrospective cohort study. Lancet 395: 1054-1062, 2020. PMID: 32171076. DOI: 10.1016/ S0140-6736(20)30566-3

6 Long LQ, Huang T, Wang YQ, Wang ZP, Liang Y, Huang TB, Zhang HY, Sun W and Wang T: COVID-19 patients' clinical characteristics, discharge rate, and fatality rate of meta-analysis. J Med Virol, 2020. PMID: 32162702. DOI: 10.1002/jmv.2575
7 Zhang JJ, Dong X, Cao YY, Yuan YD, Yang YB, Yan YQ, Akdis $\mathrm{CA}$, and Gao YD: Clinical characteristics of 140 patients infected with SARS-CoV-2 in Wuhan, China. Allergy Feb 75(7): 1730-1741, 2020. PMID: 32077115. DOI: 10.1111/all.14238

8 Ministero della Salute, Direzione Generale della Prevenzione Sanitaria, Circolare Prot. $N^{\circ}$ 0001997-22/01/2020-DGPREDGPRE-P del 22 gennaio 2020 All 3 "Polmonite da nuovo coronavirus (2019-nCov) in Cina" Available at: http:// www.trovanorme.salute.gov.it/norme/renderNormsanPdf?a nno $=2020 \&$ codLeg $=72796 \&$ parte $=1 \% 20 \&$ serie $=$ null $\quad$ [Last accessed on December 10 2020]

9 Ministero della Salute, Direzione Generale della Prevenzione Sanitaria, Aggiornamento Circolare polmonite 2019-nCoV Prot.N ${ }^{\circ}$ 0002302-27/01/2020-DGPRE-DGPRE-P All 1 (A01) del 27/01/2020 "Definizione di caso per la segnalazione". Available at: http://www.trovanorme.salute.gov.it/norme/renderNormsan Pdf?ann o=2020\&codLeg $=72847 \&$ parte $=1 \% 20 \&$ serie $=$ null [Last accessed on April 30 2020]

10 Ministero della Salute, Direzione Generale della Prevenzione Sanitaria, Aggiornamento Circolare polmonite 2019-nCoV Prot.N ${ }^{\circ}$ 0002302-27/01/2020-DGPRE-DGPRE-P All 2 (A02) del 27/01/2020 "Diagnostica di Laboratorio". Available at: http://www.trovanorme.salute.gov.it/norme/renderNormsanPdf?a nno $=2020 \& \operatorname{codLeg}=72847 \&$ parte $=1 \% 20 \&$ serie $=$ null [Last accessed on April 30 2020]

$11 \mathrm{WHO}$ - Rational use of personal protective equipment for coronavirus disease 2019 (COVID-19). Available at: https://apps.who.int/iris/bitstream/handle/10665/331215/WHO2019-nCov-IPCPPE_use-2020.1-eng.pdf [Last accessed on April 30 2020]

12 Siegel JD, Rhinehart E, Jackson M, Chiarello L and the Healthcare Infection Control Practices Advisory Committee 2007 Guideline for Isolation Precautions: Preventing transmission of infectious agents in healthcare settings. Available at: https://www.cdc.gov/infectioncontrol/guidelines/isolation/ index.ht $\mathrm{ml}$ [Last accessed on April 30 2020]

13 WHO - Guidelines on Hand Hygiene in Health Care, April 2009. Available at: https://www.ncbi.nlm.nih.gov/books/NBK144013 [Last accessed on June 26 2020]

14 Casanova L, Alfano-Sobsey E, Rutala WA, Weber DJ and Sobsey M: Virus transfer from personal protective equipment to healthcare employees' skin and clothing. Emerg Infect Dis 14(8): 1291-1293, 2008. DOI: 10.3201/eid1408.080085

15 Un tablet per i pazienti: la tecnologia "invade" l'ospedale San Martino. Available at: https://www.genova24.it/2017/03/untablet-pazienti-la-tecnologia-invade-lospedale-san-martino177395 [Last accessed on April 30 2020]

16 COVID 19: Elective case triage guidelines for surgical care: Breast cancer surgery. Available at: https://www.facs.org/covid19/clinical-guidance/elective-case/breast-cancer [Last accessed on April 30 2020]

17 Liu Q, Luo D, Cai S, Li Q and Li X: P-TNM staging system for colon cancer: Combination of P-stage and AJCC TNM staging system for improving prognostic prediction and clinical management. Cancer Manag Res 10: 2303-2314, 2018. PMID: 30104899. DOI: $10.2147 /$ CMAR.S165188

18 Linee Guida AIOM: "Tumori del colon". October, 2019. Available at: https://www.aiom.it/wp-content/uploads/2019/10/ 2019_LG_AIOM_Colon-1.pdf [Last accessed on June 26 2020] 
19 Linee Guida AIOM "Neoplasie del retto e ano". October, 2019 Available at: https://www.aiom.it/wp-content/uploads/2019/10/ 2019_LG_AIOM_Retto_ano.pdf [Last accessed on June 26 2020]

20 Spinelli A and Pellino G: COVID-19 pandemic: Perspectives on an unfolding crisis. Br J Surg 107(7): 785-787, 2020. PMID: 32191340. DOI: 10.1002/bjs.11627

21 Bujko K, Partycki M and Pietrzak L: Neoadjuvant radiotherapy (5×5 Gy): Immediate versus delayed surgery. Recent Results Cancer Res 203: 171-187, 2014. PMID: 25103005. DOI: 10.1007/978-3-319-08060-4_12

22 De Felice F and Petrucciani N: Treatment approach in locally advanced rectal cancer during coronavirus (COVID-19) pandemic: Long course or short course? Colorectal Dis 22(6): 642-643, 2020. PMID: 32237263. DOI: 10.1111/codi.15058

23 Lefèvre JH, Mineur L, Cachanado M, Denost Q, Rouanet P, de Chaisemartin C, Meunier B, Mehrdad J, Cotte E, Desrame J, Karoui M, Benoist S, Kirzin S, Berger A, Panis Y, Piessen G, Saudemont A, Prudhomme M, Peschaud F, Dubois A, Loriau J, Tuech JJ, Meurette G, Lupinacci R, Goasguen N, Creavin B, Simon T, Parc Y and The French Research Group of Rectal Cancer Surgery (GRECCAR): Does a longer waiting period after neoadjuvant radiochemotherapy improve the oncological prognosis of rectal cancer? Three years' follow-up results of the Greccar-6 randomized multicenter trial. Ann Surg 270(5): 747-754, 2019. PMID: 31634178. DOI: $10.1097 /$ SLA.0000000000003530
24 Lefevre JH, Mineur L, Kotti S, Rullier E, Rouanet P, de Chaisemartin C, Meunier B, Mehrdad J, Cotte E, Desrame J, Karoui M, Benoist S, Kirzin S, Berger A, Panis Y, Piessen G, Saudemont A, Prudhomme M, Peschaud F, Dubois A, Loriau J, Tuech JJ, Meurette G, Lupinacci R, Goasgen N, Parc Y, Simon $\mathrm{T}$ and Tiret E: Effect of interval ( 7 or 11 weeks) between neoadjuvant radiochemotherapy and surgery on complete pathologic response in rectal cancer: A multicenter, randomized, controlled trial (GRECCAR-6). J Clin Oncol 34(31): 3773-3780, 2016. DOI: $10.1200 /$ JCO.2016.67.6049

25 Fregatti P, Gipponi M, Giacchino M, Sparavigna M, Murelli F, Toni ML, Calabrò MT, Orsino L and Friedman D: Breast Cancer Surgery During the COVID-19 Pandemic: An Observational Clinical Study of the Breast Surgery Clinic at Ospedale Policlinico San Martino - Genoa, Italy. In Vivo 34(3 Suppl): 1667-1673, 2020. PMID: 32503827. DOI: 10.21873/invivo.11959

Received October 27, 2020

Revised December 3, 2020

Accepted December 11, 2020 